\title{
Induction of morphogenesis in the callus culture of Lavandula angustifolia Mill.
}

\author{
Natalia Yegorova $^{1 *}$, Natalia Kruglova $^{2}$, Ilshat Galin ${ }^{2}$, and Irina Stavtzeva ${ }^{1}$ \\ ${ }^{1}$ FSBSI "Research Institute of Agriculture of Crimea", 295493, Simferopol, Kievskaya str.150, Russia \\ ${ }^{2}$ Ufa Institute of biology of UFRC RAS, 450054, Ufa, pr. Oktyabrya, 69, Russia
}

\begin{abstract}
The influence of the nutrient medium hormonal composition on the induction of calluso- and morphogenesis in Lavandula angustifolia Mill., as well as the content of some endogenous hormones (indolylacetic acid, cytokinins, abscisic acid) in morphogenic and non-morphogenic calli, were studied. The leaf explants were isolated from obtained in vitro plants of the Stepnaya and Vdala cultivars. In primary calli with a frequency of $5.7-11.6 \%$, buds and shoots were formed on different Murashige and Skoog (MS) culture media supplemented with 6-benzylaminopurine, thidiazuron, kinetin, and gibberellic acid. In the callus of the first passage in both cultivars, the induction of morphogenesis was observed with the greatest frequency (39.5-43.2\%) on the MS medium with the addition of $1.0 \mathrm{mg} / 1$ 6-benzylaminopurine and $1.0 \mathrm{mg} / \mathrm{l} \mathrm{kinetin.} \mathrm{Morphogenic} \mathrm{calli} \mathrm{of}$ the Vdala cultivar were characterized by a higher content of cytokinins, but a lower content of indolylacetic and abscisic acids compared with nonmorphogenic ones.
\end{abstract}

\section{Introduction}

Modern biotechnological techniques, based on the use of callus cultures, allow you to create and accelerate the propagation of valuable plant genotypes, obtain secondary metabolites in vitro and solve many problems that arise when using traditional breeding methods. At the same time, calli cultivated in isolated conditions are unique experimental systems for studying the mechanisms of plant morphogenesis. To date, studies of various aspects of callusogenesis in vitro were carried out using many plant species as examples. It was established that the induction of calluso- and morphogenesis was determined by a complex of interrelated endogenous and exogenous factors [1-3].

Lavandula angustifolia Mill. is one of the most common essential oil plants in Southern Russia. Lavender oil is used in the food, perfumery and cosmetic industries, ceramic, paint and varnish production and especially in various branches of medicine [4].

Biotechnological studies of lavender are more often related to the optimization of clonal micropropagation methods using direct morphogenesis in culture of meristems or stem segments in vitro $[5,6]$. Less actively are developed methods of creation of new genotypes for breeding using somaclonal variation, mutagenesis and cell selection in vitro [7]. The

\footnotetext{
* Corresponding author: yegorova.na@mail.ru
} 
most important stage of such biotechnologies is the induction of morphogenesis in callus cultures and the regeneration of plants. A few studies in this area are mainly aimed at optimizing the composition of culture media [8-11]. Of course, it is necessary to improve methods to increase the frequency of induction of morphogenesis in calli (by optimizing the explant type, the composition of culture media, in vitro cultivation conditions, etc.). However, it is especially important to identify the mechanisms of induction and regulation of morphogenesis pathways leading to the formation of regenerants. Particular attention should be paid to the study of hormonal regulation of these processes for more complete use of the regeneration potential of callus cells since hormones are the key factors in plant morphogenesis in vivo and in vitro.

One of the directions in the development of plant biotechnologies using calli is the identification of the relationship between the content of endogenous (in explant or callus) and the concentration of exogenous (in a culture medium) hormones for regulating morphogenesis pathways in vitro. Such studies are not numerous and they were carried mainly on cereals [12-14]. Based on the available literature, studies of endogenous hormones accumulation in order to identify their participation in the callusogenesis and the regulation of morphogenesis in callus cultures in vitro for lavender have not been previously conducted.

The purpose of this work was to study the influence of the hormonal composition of the culture medium on the induction of callus and morphogenesis, as well as to analyze the content of endogenous hormones in calli connected to the induction of in vitro morphogenesis in Lavandula angustifolia.

\section{Materials and Methods}

Cultivars of Lavandula angustifolia Mill. Vdala and Stepnaya were the material for this research. As explants for the induction of callusogenesis, the leaves of plants obtained in vitro from meristems ( $4^{\text {th }}$ subculture) were used. The explants were cultured on the different modifications of Murashige and Skoog (MS) culture media, supplemented with $\alpha$ naphthylacetic acid (NAA), 6-benzylaminopurine (BAP), kinetin (Kin), thidiazuron (TDZ), and gibberellic acid $\left(\mathrm{GA}_{3}\right)$ (Sigma, USA). The cultivation was carried out in test tubes with $10 \mathrm{ml}$ of agar medium at $24-26^{\circ} \mathrm{C}, 70 \%$ of relative air humidity, under 16 -h photoperiod with light intensity 2000-3000 lux.

Calli were subcultivated every 30-35 days. The mass of the callus transplant was 90-100 $\mathrm{mg}$. At the end of the growing cycle, the frequency of callusogenesis induction (the number of explants with callus, \% of their total number) was determined. The frequency of morphogenesis was also analyzed as the number of calli with buds and shoots as a percentage of the total number of explants (for primary callus) or as a percentage of the number of calli (for callus of the first passage).

To determine the endogenous hormones (cytokinins, indolylacetic acid and abscisic acid), non-morphogenic and morphogenic calli of the Vdala cultivar were used. For analysis, calli of the $4^{\text {th }}$ passage at the stationary phase of the growth cycle (30 days of cultivation) were taken. Calli were homogenized, extracted with $80 \%$ ethanol and incubated at $4^{\circ} \mathrm{C}$ during $12 \mathrm{~h}$. The homogenate was filtered; the filtrate was evaporated to aqueous residues. The quantitative content of hormones was tested by the enzyme immunoassay method adapted by authors to the calli study [14].

In this study, the equipment provided by the Agidel Collective Use Center of UFRC RAS and by the Laboratory of Biotechnology FSBSI "Research Institute of Agriculture of Crimea" was used.

In vitro tissue culture experiments were carried out in three replications; 20 explants were analyzed at each variant. Statistical processing of the obtained data was carried out 
using the Microsoft Office Excel 2010, USA. The significance of differences was determined using the t-test at $\mathrm{P} \leq 0.05$. The tables show average values and their standard errors.

\section{Results and Discussion}

Previously, for some cultivars of L. angustifolia, we have optimized culture media for the induction of calluso- and morphogenesis from leaf explants isolated from greenhouse plants. Morphogenic calli with a frequency of up to $26.8-31.0 \%$ were formed on MS media with the addition of BAP or kinetin [15]. In the present work, the possibility of using leaves isolated from obtained in vitro plants as explants was studied. Loose, light-beige, sometimes with green areas calli were formed on the surface of explants after 3-4 weeks of cultivation. The frequency of callus formation depended on the hormonal composition of the medium and cultivar (Table 1). The maximum frequency of callusogenesis (up to $100 \%$ ) in both cultivars was noted on the medium MS160 (containing NAA and BAP) previously recommended by us for lavender [15]. On this medium, well-proliferating calli with a mass of more than $600-800 \mathrm{mg}$ were usually formed. On other media, containing cytokinins and $\mathrm{GA}_{3}$, the frequency of callusogenesis reached $72.3-77.7 \%$. In these cases, small dense calli with a mass of not more than 200-300 $\mathrm{mg}$ were formed.

In order to analyze the induction of morphogenesis in callus cultures of lavender, culture media containing cytokinins (BAP and kinetin) were tested. Also, we studied the effect the synthetic growth regulator thidiazuron (MS554 and MS556 media), which has both cytokinin and auxin effects, on the explant development. It was established that on some media in the primary calli were formed green dense morphogenic areas, from which the buds were developed. The number of explants with such morphogenic calli in the Vdala cultivar was less (up to 5.7\%) than in the Stepnaya (up to $11.6 \%$ ). In the Vdala cultivar, the induction of morphogenesis in callus cultures was revealed only on the two modifications of MS medium containing kinetin and BAP (MS552) or thidiazuron and $\mathrm{GA}_{3}$ (MS556). At the same time, in the cv. Stepnaya, morphogenic calli formed on a wider range of media. These media contained different hormones such as BAP (MS549 and MS550), kinetin or BAP together with $\mathrm{GA}_{3}$ (MS546, MS551), TDZ (MS554, MS556), BAP and kinetin (MS552).

In the first passage, after transferring calli from MS160 medium to the medium with BAP or TDZ, no buds formation was observed. An analysis of callus cultivations on the nine media showed that the largest number of morphogenic calli in the Stepnaya and Vdala cultivars (39.5 and 43.2\%, respectively) was noted on MS552 medium supplemented with $1.0 \mathrm{mg} / \mathrm{l} \mathrm{kinetin}$ and $1.0 \mathrm{mg} / \mathrm{l} \mathrm{BAP}$. After subculturing calli on those nutrient media where bud induction in the primary callus has already been noted, a significant increase in the frequency of morphogenesis compared with primary callus was revealed. So, in the Vdala cultivar on MS552 medium, in the primary callus, the morphogenesis frequency was $2.5 \%$, and in the callus of the first passage $-43.2 \%$ (Table 1). Such an increase in the number of morphogenic calli at the first passage may indicate that the formation of meristematic cell complexes began in primary calli, but only after subculture the buds (gemmogenezis in vitro) and then shoots developed from these complexes.

It should be noted that on some culture media, the cultivation of calli during one passage was not enough for the development of buds and shoots. For example, in the Vdala cultivar in primary callus on MS media with BAP (MS549 and MS551), morphogenesis was not observed (Table 1). However, further passages on the same media led to the formation of buds in 5.7 and $32.5 \%$ of calli. 
Table 1. Influence of culture medium composition and cultivar on the induction of calluso- and morphogenesis in Lavandula angustifolia in vitro

\begin{tabular}{|c|c|c|c|c|c|c|}
\hline \multirow[t]{2}{*}{ Cultivar } & \multicolumn{2}{|c|}{$\begin{array}{l}\text { Culture medium for obtaining } \\
\text { primary callus }\end{array}$} & \multicolumn{2}{|c|}{$\begin{array}{l}\text { The frequency of } \\
\text { induction, \% of the } \\
\text { number of explants }\end{array}$} & \multirow{2}{*}{$\begin{array}{l}\text { Nutrient } \\
\text { medium in } \\
\text { the first } \\
\text { passage }\end{array}$} & \multirow{2}{*}{$\begin{array}{l}\text { Frequency } \\
\text { of } \\
\text { morphogene } \\
\text { sis in the } \\
\text { first } \\
\text { passage, \% }\end{array}$} \\
\hline & medium & $\begin{array}{c}\text { hormonal } \\
\text { supplements in a MS } \\
\text { medium, }(\mathrm{mg} / \mathrm{l})\end{array}$ & $\begin{array}{l}\text { callusog } \\
\text { enesis }\end{array}$ & $\begin{array}{l}\text { indirect } \\
\text { morphoge } \\
\text { nesis }\end{array}$ & & \\
\hline \multirow[t]{12}{*}{ Vdala } & \multirow[t]{2}{*}{ MS160 } & \multirow[t]{2}{*}{ NAA (1.0)+BAP(0.5) } & \multirow[t]{2}{*}{$97.6 \pm 8.6$} & \multirow[t]{2}{*}{0} & MS549 & 0 \\
\hline & & & & & MS554 & 0 \\
\hline & MS544 & Kin (1.0) & $56.5 \pm 7.0$ & 0 & MS552 & 0 \\
\hline & \multirow[t]{2}{*}{ MS546 } & \multirow[t]{2}{*}{$\operatorname{Kin}(1.0)+\mathrm{GA}_{3}(0.5)$} & \multirow[t]{2}{*}{$63.4 \pm 5.4$} & \multirow[t]{2}{*}{0} & MS546 & 0 \\
\hline & & & & & MS552 & 0 \\
\hline & MS549 & $\mathrm{BAP}(1.0)$ & $62.9 \pm 7.2$ & 0 & MS549 & $5.7 \pm 0.4$ \\
\hline & MS550 & $\operatorname{BAP}(2.0)$ & $66.7 \pm 7.0$ & 0 & MS550 & $32.5 \pm 3.7$ \\
\hline & \multirow[t]{2}{*}{ MS551 } & \multirow[t]{2}{*}{$\operatorname{BAP}(1.0)+\mathrm{GA}_{3}(0.5)$} & \multirow[t]{2}{*}{$58.8 \pm 6.5$} & \multirow[t]{2}{*}{0} & MS552 & $20.0 \pm 1.9$ \\
\hline & & & & & MS550 & 0 \\
\hline & MS552 & Kin $(1.0)+$ BAP $(1.0)$ & $65.5 \pm 6.2$ & $2.5 \pm 0.9$ & MS552 & $43.2 \pm 4.5$ \\
\hline & MS554 & TDZ (1.0) & $77.7 \pm 7.2$ & 0 & MS554 & 0 \\
\hline & MS556 & $\mathrm{TDZ}(1.0)+\mathrm{GA}_{3}(0.5)$ & $73.5 \pm 7.1$ & $5.7 \pm 1.2$ & MS556 & $9.7 \pm 1.1$ \\
\hline \multirow[t]{12}{*}{ Stepnaya } & \multirow[t]{2}{*}{ MS160 } & \multirow[t]{2}{*}{ NAA $(1.0)+$ BAP $(0.5)$} & \multirow[t]{2}{*}{100.0} & \multirow[t]{2}{*}{0} & MS549 & 0 \\
\hline & & & & & MS554 & 0 \\
\hline & MS544 & Kin (1.0) & $48.5 \pm 52$ & 0 & MS544 & 0 \\
\hline & MS546 & $\operatorname{Kin}(1.0)+\mathrm{GA}_{3}(0.5)$ & $59.7 \pm 62$ & $2.2 \pm 1.0$ & MS546 & $18.5 \pm 2.0$ \\
\hline & \multirow[t]{2}{*}{ MS549 } & \multirow{2}{*}{$\operatorname{BAP}(1.0)$} & \multirow[t]{2}{*}{$68.8 \pm 6.7$} & \multirow[t]{2}{*}{$1.5 \pm 0.8$} & MS549 & $20.0 \pm 2.1$ \\
\hline & & & & & MS550 & 0 \\
\hline & MS550 & $\mathrm{BAP}(2.0)$ & $67.6 \pm 8.4$ & $11.6 \pm 1.7$ & MS550 & $18.5 \pm 2.0$ \\
\hline & MS551 & $\operatorname{BAP}(1.0)+\mathrm{GA}_{3}(0.5)$ & $71.5 \pm 6.8$ & $10.8 \pm 12$ & MS551 & $15.6 \pm 13$ \\
\hline & MS552 & Kin (1.0)+ BAP (1.0) & $72.3 \pm 7.8$ & $2.2 \pm 1.0$ & MS552 & $39.5 \pm 3.4$ \\
\hline & \multirow[t]{2}{*}{ MS554 } & \multirow[t]{2}{*}{ TDZ (1.0) } & \multirow[t]{2}{*}{$56.7 \pm 6.2$} & \multirow[t]{2}{*}{$3.5 \pm 0.6$} & MS554 & $9.7 \pm 1.3$ \\
\hline & & & & & MS556 & $6.8 \pm 0.9$ \\
\hline & MS556 & $\mathrm{TDZ}(1.0)+\mathrm{GA}_{3}(0.5)$ & $65.0 \pm 6.2$ & $5.8 \pm 0.8$ & MS556 & $28.8 \pm 2.1$ \\
\hline
\end{tabular}

Earlier, to obtain morphogenic callus cultures in different lavender species, leaves [7-9, 16], stems [17], shoot tips [10,11], and meristems [18] were used. Explants were usually isolated from plants grown in field or greenhouse conditions. The results of research presented in our work for the first time showed the possibility of using leaves of lavender plants obtained in meristem culture in vitro as an explants for calluso- and morphogenesis induction.

Literature data on the composition of "morphogenic" media for the cultivation of various lavender explants are very contradictory. In some species of lavender for the induction of morphogenesis in calli, BAP [17], or this cytokinin in combination with the IBA [10] were used. The maximum frequency of the formation of buds and shoots from callus obtained from $L$. angustifolia shoot tips was revealed on MS medium with BAP (1.0 $\mathrm{mg} / \mathrm{l})$, kinetin $(1.0 \mathrm{mg} / \mathrm{l})$, and NAA $(0.5 \mathrm{mg} / \mathrm{l})$ [11]. It was reported about the effectiveness of the introduction in the culture medium of kinetin in combination with NAA and IBA for the buds and shoots induction in callus cultures [16]. There is evidence of obtaining multiple shoots from L. angustifolia callus on a culture medium with TDZ [9].

In our experiments, for two L. angustifolia cultivars, the possibility of morphogenesis induction on media with the addition of different cytokinins (kinetin, BAP, TDZ) was shown. The maximum frequency of morphogenic callus formation in the first passage in both cultivars (39.5-43.2\%) was detected on MS medium supplemented with BAP and kinetin $(1.0 \mathrm{mg} / \mathrm{l} \mathrm{each})$. 
An important direction of experimental research of callus cultures is the analysis of endogenous hormones content in them, which can affect many morphogenetic processes in vitro. The content of endogenous hormones was determined on the example of nonmorphogenic and morphogenic calli of Vdala cultivar. Non-morphogenic calli (obtained on MS160 medium) were homogeneous, friable, light beige and did not have visually visible morphogenic structures. Morphogenic calli (obtained on MS549 medium) had dense green areas with buds, later from this buds developed microshoots. The calli were cultured on the medium of the same composition (MS160) for three passages to reduce the influence of exogenous hormones. It was established that different types of calli varied significantly in the content of the studied endogenous phytohormones (Table 2). In morphogenic calli, the total content of cytokinins was 4.7 times higher than in non-morphogenic ones. This was mainly due to an increase in the content of zeatin riboside and zeatin nucleotide. The morphogenic calli showed the reduction in the content of indolylacetic acid (almost 6 times) and abscisic acid (twice) compared with non-morphogenic calli.

Table 2. The content of phytohormones in various types of calli in lavender cultivar Vdala (ng/g wet weight)

\begin{tabular}{|c|c|c|}
\hline Phytohormone & Morphogenic callus & Non-morphogenic callus \\
\hline Zeatin riboside & $8.2 \pm 1.3$ & $0.5 \pm 0.1^{*}$ \\
\hline Zeatin nucleotide & $6.6 \pm 0.4$ & $1.9 \pm 0.3^{*}$ \\
\hline Zeatin & $1.6 \pm 0.3$ & $1.1 \pm 0.1$ \\
\hline Summer of cytokinins & $16.4 \pm 2.0$ & $3.6 \pm 0.5^{*}$ \\
\hline Indolylacetic acid & $10.5 \pm 0.6$ & $64.1 \pm 5.2^{*}$ \\
\hline Abscisic acid & $0.5 \pm 0.1$ & $1.0 \pm 0.1^{*}$ \\
\hline
\end{tabular}

* Differences between non-morphogenic and morphogenic callus are significant at $\mathrm{P} \leq 0.05$

It is known that the restructuring of undifferentiated callus cells into organized structures during different pathways of morphogenesis in vitro is accompanied by molecular genetic, physiological, biochemical, cytohistological and morphological changes $[1,2]$.

The ability of calli to develop along the certain path of morphogenesis is largely due to the balance between the content of endogenous hormones in the callus and the concentration of exogenous hormones in the culture medium. This has been revealed, for example, in some cereals $[12,14,19]$. So, for wheat and barley, it was found that the ability to somatic embryogenesis in vitro was determined by the ratio of IAA:ABA content in calli, with rather similar cytokinins content in both embryogenic and non-embryogenic calli types [14]. In our work, it was shown that in the morphogenic lavender calli, the content of cytokinins (zeatin riboside and zeatin nucleotide) increased and the content of ABA and IAA decreased. Such differences in the data obtained, apparently, may be due not only to the characteristics of plant species and explants used (embryos/leaves) but mostly ways of morphogenesis in calli (somatic embryogenesis/gemmogenesis).

Thus, as a result of the studies, the features of the influence of the hormonal composition of the culture medium on the induction of morphogenesis in calli obtained from the leaves of test tube plants of L. angustifolia were revealed. It was shown that in primary calli with a frequency of up to $5.7-11.6 \%$, buds and shoots were formed on MS media supplemented with BAP, TDZ, kinetin, $\mathrm{GA}_{3}$. In the Stepnaya cultivar, morphogenic calli formed on a wider range of media than in the Vdala. In the calli of the first passage in both cultivars, the induction of morphogenesis was observed with the greatest frequency (39.5-43.2\%) on the MS medium with the addition of $1.0 \mathrm{mg} / \mathrm{l} \mathrm{BAP}$ and $1.0 \mathrm{mg} / \mathrm{l} \mathrm{kinetin}$. Significant differences in the Vdala cultivar were revealed in the content of endogenous hormones in morphogenic and non-morphogenic calli in the fourth passage. Morphogenic 
calli were characterized by a higher content of cytokinins, but a lower content of IAA and ABA compared with non-morphogenic ones.

The authors express their sincere gratitude to Prof. G.R. Kudoyarova for valuable advices during the research and discussion of the results.

\section{References}

1. M. Ikeuchi, D.S. Favero, Y. Sakamoto, A. Iwase, D. Coleman, B. Rymen, K. Sugimoto, Annu. Rev. Plant Biol., 70, 377-406 (2019)

2. N.N. Kruglova, G.E. Titova, O.A. Seldimirova, Russ. J. Dev. Biol., 49, 245-259 (2018

3. A. Feher, Front. Plant Sci., 10, 536 (2019)

4. V.S. Pashtetskii, N.V. Nevkrytaya, A.V. Mishnev, L.G. Nazarenko Essential oil industry in the Crimea. Yesterday, today, tomorrow (Arial, Simferopol, 2018).

5. S. Mokhtarzadeh, B. Demirci, H.G. Agalar, K.M. Khawar, N. Kirimer, Rec. Nat. Prod., 13, 121-128 (2019).

6. N.A. Yegorova, I.V. Mitrofanova, V.A. Brailko, O.A. Grebennikova, A.E. Paliy, I.V. Stavtseva, Russ. J. Plant Physiol., 66, 326-334 (2019)

7. N.A. Yegorova, Fiziol. Rast. Genet., 46, 108-120, (2014).

8. S. Kintzios, I. Papanastasiou, P. Tourgelis, Ch. Papastellatos, V. Georgopoulos, J. Drossopoulos, J. Herbs, Spices Med. Plants, 9 (2-3), 223-227 (2002).

9. L. Falk, R. Biswas, A. Boeckelmann, A. Lane, S.S. Mahmoud, J. Essent. Oil Res., 21, 225-228 (2009).

10. V. Parkash, H. Singh, International Journal of Agricultural Technology (IJAT), 9, 691-702 (2013).

11. D. Leelavathi, M.V. Govinda Raju, Yashoda, Narendra Kuppan, J. Pharm. Biol., 4, 66-70 (2014).

12. H. Hisano, T. Matsuura, I.C. Mori, M. Yamane, K. Sato, Plant Physiol. Bioch., 99, 6672 (2016)

13. J.K. Hong, K.J. Park, G.-S. Lee, D.Y. Kim, J.K. Kim, S.B. Lee, E.J. Suh, Y.H. Lee, J. Plant Biotechnol., 44, 49-55 (2017)

14. O.A. Seldimirova, G.R. Kudoyarova, N.N. Kruglova, I.R. Galin, D.S. Veselov, Russ. J. Dev. Biol., 50, 124-135, (2019)

15. N.A. Yegorova, Obtaining of plant-regenerants in callus culture and micropropagation in vitro of lavender (methodical recommendations) (Simferopol, IELR UAAN, 2008).

16. N. Jahan, R. Mustafa, M.A. Zaidi, A. Mansoor, J. Khan, D. Baluch, Curr. Res. J. Biol. Sci., 4, 258-260 (2012).

17. G. Ghiorghita, D.E. Maftei, D. Nicuta, Propag. Ornam. Plants, 9, 47-49 (2009).

18. T.S. Sabzevar, R.A. Ghavidel, S. Foroghian, J. Pharm. Pharmacol., 3, 338-344 (2015).

19. Sh.-T. Lee, W.-L. Huang, Bot. Stud., 54, (2013) 